\title{
植物生理生态学的学科起源与发展史
}

\author{
蒋高明 \\ (中国科学院植物研究所植被数量生态学重点实验室, 北京 100093)
}

\begin{abstract}
摘 要 探讨了植物生理生态学的起源及其发展问题。植物生理生态学是植物生态学的一个分支, 它主要是用生 理的观点和方法来分析生态学现象。从现有的资料看, 该门学科的创始人当为德国学者辛泊尔 (A. F. W. Schimper) ;但近代最早建议将植物生理生态学作为独立的学科的为比林斯 (W. D. Billings) 拉夏埃尔 (W. Larcher) 为该门 学科在国际上的传播起到了非常重要的作用。在中国 近代植物生理生态学的发展应归功于李继侗先生, 然而, 李 博先生对拉夏埃尔《植物生理生态学》的翻译出版促进了该门学科在中国的传播。植物生理生态学目前在国内外 发展十分迅速, 由于它能够对一些生态学现象以及资源的可持续利用给以机理上的解释, 因此越来越受到研究者 的重视。
\end{abstract}

关键词 植物生理生态学 学科 起源 发展

\section{ON THE ORIGIN AND DEVELOPMENT OF ECOLOGICAL PLANT PHYSIOLOGY}

\author{
JIANG Gao-Ming \\ (Laboratory of Quantitative Vegetation Ecology , Institute of Botany , Chinese Academy of Sciences , Beijing 100093 , China)
}

\begin{abstract}
The origin and development of ecological plant physiology is discussed. An important subject of plant ecology , ecological plant physiology probes the mechanisms of an ecological phenomenon through physiological theory and technology. According to the available historical information, A. F. W. Schimper, a German sophist, can be regarded as the father of this subject. However, the person who suggested that ecological plant physiology be treated as an independent field of ecology was W. D. Billings. Professor W. Larcher has contributed greatly to the promulgation of the subject throughout the world. In China , the modern development of ecological plant physiology was due to Professor T. T. Li. However, the translation and promulgation of Larcher' s works was done by Professor B. Li , who popularized this subject in the ecological field of China. Nowadays, the study of ecological plant physiology has expanded rapidly both overseas and in China, largely owing to the fact that it can explain ecological processes on the basis of eco-physiological mechanisms and help guide the sustainable use of natural resources. For Chinese researchers, hot topics such as the response of plants to environmental stresses that grow on the Tibetan Plateau, the response of plants to $\mathrm{CO} 2$ enriched environments , and plant responses to restoration efforts of human degraded ecosystems should be of particular interest.
\end{abstract}

Key words Ecological plant physiology, Subject, Origin , Development

植物生理生态学 (Ecological plant physiology) 和 植物生态生理学 (Plant ecophysiology) 讨论的问题有 很大的一致性, 甚至在一些场合下, 它们被混同为同 样的学科。造成同一学科的不同理解起因于中国学 者对外文术语的翻译与理解。例如, 奥地利学者 Larcher 编著的 Ecological Plant Physiology (原书以德 文出版)，李博等在英译本的基础上翻译为《植物生 理生态学》(Larcher，1980)，17 年后同是该本书(再 版) 翟志席等就译成《植物生态生理学》( Larcher, 1997)了。这给初学者带来了困惑。实际上, 植物生 理生态学与植物生态生理学中 生态” 乌 生理” 前后
排列的差异, 意味着研究所强调的重点有所不同, 研 究生理的往往习惯用 生态生理”, 它或多或少地涉 及机理的变化和反响;而 生理生态”研究多限于某 种生理现象而较少涉及机理。尽管如此, 两门学科 在大多场合下是可以互相包容的。与植物生理生态 学的关系比较密切的学科还有植物生态生理学 (Plant ecophysiology)、实验植物学 (Experimental botany)、环境植物学 (Environmental botany)、植物进化 生态学 (Plant evolutionary ecology)、功能生态学 (Functional ecology) 等。它们基本上研究相似的问题, 但 从中文的译法以及 约定俗成” 与 先入为主” 的实用 
角度出发, 在中文里我们接受 植物生理生态学”这 一术语作为这一学科的正式名称, 避免形成更大的 混乱。

\section{1 植物生理生态学的特点}

植物生理生态学是植物生态学的一个分支, 它 主要是用生理的观点和方法来分析生态学现象。因 此它研究生态因子以及植物生理现象之间的关系, 即生态学与生理学的结合。植物生理生态学研究的 问题包括:1) 植物与环境的相互作用和基本机制; 2) 植物的生命过程 3) 环境因素影响下的植物代谢 作用和能量转换; 4) 有机体适应环境因子改变的能 力。进行植物生理生态学研究可以在不同的尺度水 平上展开，从分子、细胞、组织、器官、个体、到种群、 群落、甚至生态系统等都可以展开有关的研究, 但研 究的焦点是有机体本身, 即无论在哪个尺度上的研 究都要围绕个体的基本性能表现 (Performance) 来进 行。个体是自然选择中以其去留使优良性状得以保 存和发展的基本单元，个体层次起承前启后的作用。 因此，植物生理生态学的学科定位是 :1) 研究种群、 群落和生态系统功能的学科,2) 宏观与微观生物学 研究的结合点 3) 个体水平以下研究结果的证明和 理解等。

从 20 世纪 60 年代开始, 国际地圈与生物圈计 划 (IGBP) 启动;1971 年 联合国环境计划署( UNEP) 启动了国际生物圈计划( IBP)的计划, 后来该计划发 展成为人与生物圈计划” (MAB)。目前参与该计划 的国家达 110 多个,分布全球各大洲。在上述 IGBP, IBP, MAB 计划的有利推动下, 植物生理生态 学得以迅速发展。近 20 年来 植物生理生态学的研 究日新月异, 其研究从细胞到生态系统各个组织层 次放大的同时, 又重新将重点集中到个体水平。研 究对象从过去的作物和常见种为主转向生物多样性 和全球变化的关键植物种类。当前植物生理生态学 研究的新动向便围绕上述问题展开, 包括植物在温 室气体浓度上升造成的全球气温上升和由它带来的 各种全球变化中的生理生态响应;植物适应和进化 的机理，对有限资源的合理利用;光、温、水、气、养分 等多种环境因子对植物影响的相互作用;对植物生 长发育的影响 植物的抗逆性潜能和植物生长过程 的动态模拟, 特殊生境下植物的生态适应机制等等。

近年来, 由于人类经济活动对生物圈干扰的不 断升级, 造成的生态环境问题越来越突出, 如全球 气候变化、生物多样性的不断丧失、环境污染的扩大
与加重等。这些环境问题的存在和引起的不良后果 引起了各国政府与科学家的广泛关注。植物生理生 态学从生理机制上探讨植物与环境的关系、物质代 谢和能量传递规律以及植物对不同环境条件的适应 性(Larcher，1997)。由于它能够给许多生态环境问 题以生理机制上的解释，因而得到日益广泛的重视。 例如，许多著名的国际刊物，如 Plant, Cell and Environment、Oecologia 、Functional Ecology、Journal of Experimental Botany 等发表植物生理生态学研究的原始 论文不断增加。如在 Oecologia 的固定栏目中, 生理 生态学 (Ecophysiology) 被列在首位。由此可以看 出 植物生理生态学越来越多地受到重视, 已成为植 物生态学领域重要的学科方向之一。

\section{2 植物生理生态学的起源与发展阶段}

植物生理生态学来源于植物生态学, 它作为一 门成熟的学科也只有几十年的历史。最初植物生理 生态学关注的内容是介绍生态学的思想, 即介绍 植 物与环境” 系统中的基本过程、作用功能的机理。作 为植物生态学，它的起源是二元的(李继侗，1958)： 一方面是由植物地理学发展而来, 起源于德国植物 地理学家洪堡 (von Humboldt) (1769 1859) (von Humboldt , 1805) ; 另一方面则来源于植物生理学, 始 于瑞士学者德堪多 (A. P. De Candolle, 1778 1848)。至于谁是植物生理生态学的奠基人, 从目 前掌握的资料看, 似为德国植物生态学家辛泊尔 (A. F. W. Schimper) (1856 1901), 他在 1898 年发 表的经典著作《基于生理学的植物地理学》一书的序 言中就强调了植物生理生态学研究的必要性 (Schimper, 1898)。从萌芽阶段开始, 植物生理生态 学已经历了 5 个阶段。

\section{1 思辩方法和准实验方法阶段 (公元前～1750)}

在古代社会生产力低下的条件下,人们只能依 靠感官进行表面观察所获得的不充分的事实, 进行 简单的逻辑推理及非逻辑的构思, 得出一些带有猜 测性的笼统的结论。国外一些文明古国在这方面贡 献很大, 如埃及的尼罗河引水灌溉农业等。这里, 我 们以中国为例, 介绍一些朴素的植物生理生态学思 想。《尚书. 洪范》(记载的时代约公元前 $2000 \sim 600$ 年)中的五行 一曰水，二曰火，三曰木，四曰金 ,五曰 土”说，从非常朴素的观点提出了万物包括生命在内 的起源及其相互关系。《尚书. 周礼》有关于阳生 植物与阴生植物的记载, 如阳木生山南者,阴木生 山北者”以及 仲冬斩阳木, 仲夏斩阴木”。春秋时期 
(公元前 $770 \sim 476$ 年) 的《管子. 地员篇》专门记载 了土壤类型特点与自然分布的和适宜种植的植物, 将土壤分为上中下 3 等, 每等又分 6 类, 每类又分 5 种, 计 90 种，所种植的谷物列有 36 种之多。如文中 记载 赤垆, 经强肥, 五种无不宜, 其麻白, 其布黄, 其 草宜白茅与灌, 其木宜赤棠”。西汉 (公元前 206 年 $\sim$ 公元 8 年) 的《淮南子》的 草木洪者为本, 而杀者 为末”以及 故食其口而百节肥, 灌其本而枝叶美, 天 地之性也”，描述了植物地上部与地下部的关系。 《盐铁论》中有关于光影响植物生长的记载, 如 茂林 之下无丰草, 大块之间无美苗”; 北魏 (420 589 年) 贾思劦著的《齐民要术》则论述了各种农作物、蔬菜、 果树、竹木的栽培, 书中所记载的旱农地区的耕作和 谷物栽培、树苗繁殖、嫁接方法, 都是对植物生长习 性的观察总结，尤其可贵的是，贾氏提倡的 顺天时， 量地力, 则用力少而成功多; 任情反道, 劳而无获” 的 思想, 在科学比较发达的今天也是符合生理生态学 思想的。唐宋之后, 人们对植物与环境的关系理解 更进一步了, 如唐代 (618 907) 刘恂《岭表录异》记 载“: 广州地熟 种麦则苗而不实”,揭示了小麦发育 需要低温阶段的现象; 北宋 (960 1127) 《东溪试茶 录》的 茶宜高山之阴, 而喜日阳之早” 是关于植物 与光照的辨证关系的记载;元代(1271～1368)王祯 《农书 粪壤篇》记载有踏肥、大粪、苗粪、草粪、火粪、 泥粪多种肥料, 同时指出绿肥在江南地区普遍使用， 以及一切禽兽毛羽亲肌之物, 最为肥泽”, 上述描述 是关于植物与土壤关系精彩的记载。然而, 需要指 出的是, 中国古代的思想家大多是从自然界那里认 知一些基本的规律, 包括生命的起源与演化, 但后来 这些知识大多被引申为做人和做官的道理, 很少像 文艺复兴以后的西方人那样沿着认知自然的道路走 下去。这就是为什么我们祖先很早的发现不能演变 成近代自然科学的主要原因。

西方人在这一漫长的阶段中，大部分时间的科 学与技术是落后于中国的。但是工业革命以后, 西 方在自然科学的道路上却领先于中国了。如在植物 生理生态学的最初阶段, 西方的科学家开始注意到 植物与环境的关系, 波义耳 (Boyle, 1661) 最早提出 了元素、化合物和土壤盐分的概念; 20 世纪初布鲁 塞尔的医生黑尔蒙特 (van Helmont) 设计了著名的柳 树实验, 试图寻找光合作用的物质来源 (Loomis, 1960）；Woodward（1699）利用液体培养技术栽培植 物，找出了植物生长需要的一些营养物质，显然这 是受到了化学家的影响; Hales (1727) 指出了空气
是植物体的组成部分, 认识到了光合作用主要物质 的来源问题。18 世纪初，一批科学家补充了范荷尔 门等人的实验，从而完善了对光合作用的发现。

\section{2 观察与描述方法的开创阶段 $(1750 \sim 1900)$}

在生态学的初创时期,生态学研究基本上停留 在描述阶段, 而生理学研究则大部分局限在实验室 内, 植物生理生态学仍未从其双亲学科中脱离出来。 在植物生理学方面, 1862 年利比希提出了著名的最 小因子定律 ( Liebig，1862）；在植物生态学方面， 1866 年海克尔提出了生态学的概念 (Haekel, 1866)。 其后,Pfeffer (1900) 在众多学者在植物与环境观察 与描述的基础上，出版了第一部《植物生理学》, 书 中内容涉及了植物的生理过程、光合作用、呼吸作 用、同化物质的分配、水分关系、矿质营养、氮同化、 植物与环境关系, 书中的有些观点影响至今。另外 值得提出的是，哈伯兰特 (Haberlandt , 1884)、辛泊尔 (Schimper，1898)、瓦尔明 (Warming，1891) 等人分别 从植物解剖学、植物地理学和植物生态学的角度出 发, 提出了植物对环境的适应性, 并围绕各自的研究 提出了一系列重要的猜测和假说, 这些成果的获得 在很大程度上是由于他们善于观察。因此, 观察是 植物生理生态学研究的一种重要方法。

相对于后来的实验方法而言，观察方法有很多 缺点和局限性: 1) 只能得到关于事物本质的某些表 面现象, 而某些现象往往时过境迁, 不能自发重现, 限制了进一步深入研究；2）只能得到事物整体综 合的表面现象, 无法了解原因。生命现象是自然界 最复杂的运动形式,生态学过程尤其复杂, 仅仅运用 观察方法远远不能解决深入的问题，必须采用实验 方法。

\section{3 实验方法阶段 (1900～1950)}

实验方法是利用仪器或控制设施有意识地控制 自然过程条件 模拟自然现象。利用环境控制技术， 在研究某种因子对植物的影响时, 控制其它环境条 件尽量不发生改变, 这样就避开了干扰因素, 突出主 要因素, 在特定条件下探索客观规律。实验方法与 观察方法的不同在于:1)改变单个因素, 保持其它因 素不变,从而判断各个因素的作用, 使研究对象以纯 粹的、更便于观察和分析的形态表现出来。如利比 希在研究影响植物生长的营养元素中, 就是采取上 述 避轻就重” 的做法 (Loomis，1980) , 其对实验生物 学影响很大 2) 实验结果能够反复再现, 重复研究。

作为植物生理学与植物生态学的交叉学科, 植 物生理生态学也是植物生态学中实验内容最强的分 
支学科。这些工作早在 20 世纪初就有人认真地做 工作了，如 Clements (1907) 研究了植物叶片能量的 平衡;Blackman 等 (1905) 根据他的实验提出了限制 光合生产的一些基本因子, 指出光合作用受到数种 因子影响时,其受限制的程度取决于供应量最少 (小)的那个因子。虽然后来发现该定律难以判断不 同因素之间是否有交叉作用, 但它对于理解植物的 生理活动仍然具有重要意义。其后, 许多学者对环 境因子对植物生长发育过程的影响进行了大量的实 验研究，如植物气孔的开张 (Briggs et al.,1920)、光 补偿点/光饱和点 (Bates \& Roeser, 1928)、 $\mathrm{CO}_{2}$ 补偿 点 $/ \mathrm{CO}_{2}$ 饱和点 (Hoover et al. , 1933)、温度(Blackman \& Matthaei，1905)，矿物质 (Briggs，1922) 对光合作 用的影响等 取得了有意义的成果。但这些研究大 部分是在室内进行的,其研究时的环境与自然环境 差别较大, 并且主要是对单个因子的影响做研究, 故 仅能从某个侧面反映植物的生理生态特性，无法表 现在自然环境中多种因素作用对植物的功能的综合 影响。鉴于这些原因，一些先驱者开始尝试把生理 学实验搬到野外去, 如苏联的马克西姆 (Maximov, 1929)和美国的 Daubenmire(1974) 等, 研究了沙漠植 物和植物群落中的植物与环境关系。这些研究促进 了植物生理生态学作为一门独立的学科的问世。20 世纪 50 年代, 比林斯 (W. D. Billings) 最早倡议把植 物生理生态学看作是一门独立的学科。对该段详细 的历史读者可以参考 Billings (1985)写的回忆录。

实验方法阶段初期, 存在的主要问题是实验方 法的缺陷, 例如在与生理活动相应的小尺度上测定 气候因子就很困难, 成为植物生理生态学发展的一 个限制因子。直到 20 世纪 60 年代才有了精确测定 植物叶温、光合有效辐射的技术; $\mathrm{CO}_{2}$ 交换和 $\mathrm{H}_{2} \mathrm{O}$ 的 现代测定技术使植物生理生态学家可以在自然条件 下对气孔活动、蒸腾和光合速率进行连续的监测。 尽管研究手段较以前有明显的改进，但距离植物生 理生态学实验研究的要求还很远。例如当时, 只能 对草本植物和木本植物的幼苗进行控制部分环境条 件的试验，并且自然环境的模拟技术也还很不够。 再如, 如何在生长室中模拟风速的涡动效应, 至今仍 然没有解决。在水生生态系统中, 光、涡流以及碳交 换的测定都还只是初步的形式。植物地下环境及植 物地下过程测定方法相当缺乏。虽然人们对植物的 气孔控制和失水方面的理解已经取得了很大进展， 但植物细胞水分关系中的有关参数, 如膨压、渗透势 的测定仍然相当困难。在植物-离子关系方面, 情况
也相似。

\section{4 理论方法与综合方法阶段 (1950～1980)}

植物生理生态学主要发展于 20 世纪下半叶, 自 然科学得到迅速的发展。在这种形势下, 作为科学 研究的工具, 运用单一的研究方法已经不能满足需 要了。研究对象和研究方法之间的关系已经发生了 根本变化, 研究方法呈现出交叉化、多元化、综合化 的发展趋势。

植物生理生态学研究方法的演变不可能再走与 其它古老学科(如物理学和化学)的同样的路。自然 科学, 甚至一些社会科学的先进的方法和理论无时 不渗透进来。20 世纪 60 年代年代以来, 植物生理 生态学研究方法开始长足发展, 特别是野外测定手 段的不断改进和计算机的广泛采用使模型方法得到 广泛的运用。精确的测定植物代谢与其微环境变化 成为可能, 也为人工气候室内自然环境的模拟奠定 了基础。如研究多种限制因素的相互作用对 $\mathrm{CO}_{2}$ 和 $\mathrm{H}_{2} \mathrm{O}$ 气体交换的影响 (Holmgren et al ., 1965) ;对 $\mathrm{C}_{3}$ 和 $\mathrm{C}_{4}$ 代谢的研究 (Kortschack et al . , 1965) 等。 Ludlow 和 Wilson (1971) 对气体进出叶片阻力的研究, Gates (1962) 对叶片能量平衡的研究, 及 Monteith (1965)的植物干物质生产_气候模型等, 奠定了定量 研究环境对植物代谢影响的理论基础。20 世纪 60 年代末以后, 植物生长模型研究进入繁荣时期 影响 较大的有农作物同化、呼吸以及蒸腾作用的系统性 模拟模型，作物生长与生产的模拟模型等。在这方 面最突出的工作要属荷兰 Wageningen 农业研究中 心 开创了用计算机来模拟农业生产和环境与植物 群落之间的相互影响的先例 (de Wit，1978)。80 年 代 植物生理生态学家又发展了建立在生物化学反 应基础上的光合作用模型及气孔调节模型 (Farquhar et al., 1980 ; Farquhar \& von Caemmerer , 1982)。 1975 年, 奥地利学者拉夏埃尔编著的《植物生理生 态学》出版 (Larcher, 1975) , 宣告了这门学科的正式 形成。

\section{5 现代植物生理生态学阶段 (1980 今)}

进入到 20 世纪 80 年代以来, 植物生理生态学 得到了长足的发展, 这在不同层次上都得以体现。 植物个体生理生态学的研究主要以农作物、经济林 木、牧草和资源植物为研究对象，研究个体的光合生 产、水分循环和抗性生理。进入 80 年代初期, 植物 群落结构与功能的研究则成为群落生理生态学研究 的核心内容。有两个重要的原因使得这门科学在近 几十年发展迅速。其一是生态环境问题的不断出 
现, 尤其是以大气中 $\mathrm{CO}_{2}$ 浓度升高为主题的全球变 化问题, 使它在解决实际问题 (气候变化、环境污染、 粮食危机等) 上有了用武之地; 其二则是技术的进 步, 便携式快速而精确的测定仪器不断推出, 可以实 现在野外自然状态下测定植物的气体交换过程、叶 绿素荧光、能量交换、水势、水分在植物体内的流动、 冠层与根系生长的分析, 各种环境控制手段的不断 完善使实验的重复性加强; 而室内稳定性同位素技 术 (Edwards \& Walker, 1983)、元素分析技术的成功 应用则给许多生态学现象和野外观测的结果以机理 性的解释。除此以外, 系统科学的原理和方法, 如系 统论、控制论、耗散结构理论、分形理论等也广泛应 用进来(常杰等, 1995)。有关新的动态, 请参考蒋 高明(2001)的综述文章。

\section{3 植物生理生态学在国内的发展}

$1920 \sim 1960$ 我国在植物生理生态学方面研究 始于 20 世纪 20 年代, 当时一些前辈如钱崇澍 (1883 ～1965)、李继侗（1897～1961) 等在国外的工作, 涉 及植物生长发育与土壤理化性状、水分的关系, 是早 期的启蒙性工作。钱崇澍与 Osterhout 合作发表的论 文 钡、锶及铈对水绵的特殊作用”, 是中国人第一次 在国外刊物上发表有关植物生理学的论文 (Chien \& Osterhout，1917)。如果钱氏的工作是植物生理学的 内容, 其工作是开创了中国植物生理学的先端, 那么 李继侗的工作经历则开创了中国植物生理生态学研 究之先河。李氏最早在英国期刊上发表的 光对光 合速率变化的瞬时效应”, 公认是对两个光系统的先 驱报告 ( Li，1929), 可见其在植物生理学领域的造 诣。抗日战争结束, 李氏转入植物生态学研究, 较早 在国内开展植物生态的调查工作。在高等植物的呼 吸代谢方面, 汤佩松先生以水稻为研究对象发现了 EMP 无氧呼吸酶系统(汤佩松等, 1956)，从而证明了 “呼吸代谢多条路线”的思想; 在营养生理方面, 罗宗 洛于 1927 年在日本《植物学杂志》上发表了题为 不 同浓度的氢离子对植物的影响”的论文 (Loo,1927)， 其后又发表了几篇矿质营养的论文。他的这些工作 是我国植物生理学者在矿质营养方面的起始标志。 在水分生理的研究中,中国学者的工作可谓世界领 先。汤佩松与物理学家王竹溪合作发表了一篇有着 深远意义的论文 (Tang \& Wang, 1941), 用热力学原 理分析了单细胞和水分的关系，如渗透压、吸水压及 膨压等, 20 年后, 类似的水势研究才出现在国际刊 物。建国以后，一些科学家对营养元素尤其是微量
元素对植物的影响方面进行了大量的有代表性的研 究(汤玉玮等, 1955 崔 ,1954) ; 论罗宗洛为首的专 家从 50 年代开始了干旱、盐碱、寒害和涝害等方面 的研究。如果把当时分散在农、林、草各学科的生理 生态工作综合来看, 也有一定的基础。但由于当时 学科范围的限制, 没有独立发展成为植物生理生态 学。如涉及作物、林木和牧草生理生态学的研究, 有 小麦、水稻等作物的抗旱性、抗寒性，杉木、杨、柳的 生长分布与环境条件的关系; 穿心莲北移后光温处 理, 促进开花结实; 罗芙木生长发育、生物碱含量与 光照强度的关系; 羊草大针茅草原群落的单叶光合 生理生态研究。

1960 1980 从生理生态学角度开展的大田光 合生理研究, 对传统的群落光合模型作了修订。以 兴安岭和长白山红松针阔混交林、内蒙古草原、亚热 带杉木针阔混交林结构与光合生产的研究也是群体 水平的工作。这些工作与 IGBP 研究中光合生产、矿 质营养元素循环的目标一致,成为群体光合生理生 态的一个特殊领域。此外, 20 世纪 70 年代开始, 我 国生态学者围绕着大气和水质污染开展了植物与环 境污染物质关系的研究, 分别就 $\mathrm{SO}_{2} 、 \mathrm{NOx} 、 \mathrm{HCl} 、 \mathrm{Hg}$ 以及 $\mathrm{Cu} 、 \mathrm{~Pb} 、 \mathrm{Cd} 、 \mathrm{Hg}$ 等重金属的吸收积累与分配及 其净化功能也进行了研究。对大气和污水排放标准 的制订，污水灌溉对作物质量的影响以及建立生物 净化带等提供了依据。

1980 1990 植物生理生态学研究零星分散, 由于实验观测仪器设备落后陈旧, 开展的植物生理 生态学研究则近乎空白。但这一阶段非常值得一提 的是, 李博先生翻译的 Larcher 的《植物生理生态 学》,为该学科在以后中国的迅速发展奠定了基础 (Larcher，1980)。这 10 年当中, 特别需要提及的是 李继侗的 植物气候组合论”一文(李继侗,1986)，该 文全面地介绍了伦德加德 (Lundegårdh) 等人的理论， 并结合实际对我国全境的植物群落和气候组合作了 比较深入的探讨, 同时开展了一些有光、温度、水分 对植物生长发育影响的研究。

1990 今 由于仪器的更新, 尤其是中国生态 系统研究网络 (CERN) 和中国生物多样性项目 (BRIM)的实施, 以中国科学院和中国林业科学研究 院为主的研究队伍购置了大量的植物生态生理仪器 (室内和野外的),已开展了不少研究。如对不同野 生植物或大田作物的光合生理、水分生理、抗性生理 方面取得了大量的进展。 


\section{4 结束语}

植物生理生态学的特点表明，它具有植物生态 学与植物生理学双亲起源的特点, 是一门明显的交 叉学科。在学科成熟过程中, 辛泊尔、比林斯、拉夏 埃尔等人的贡献是不可以磨灭的，李继侗、李博的工 作促进了该学科在中国的传播与发展。衡量一门学 科是否有生命力, 主要是看它能否在实践中接受检 验, 能否为社会、经济或环境的可持续发展提供一种 思维的方法与研究的手段。植物生理生态学最近的 迅速发展说明, 它能够对一些生态学现象以及资源 的可持续利用给予机理上的解释, 受到了国内外学 者越来越多的重视。当然, 这门学科的交叉特点使 得在某些场合下难以区分学者们所做的工作是属于 植物生理学的还是植物生态学的, 但有一点是可以 肯定的, 即包括了生理学的严谨实验又包括了生态 学的宏观思路的研究, 在一定程度上为开展研究提 供了新思路。今后我国的植物生理生态学研究，一 是要紧紧抓住我们中国自己的生态问题，如由人类 活动引起的退化生态系统的恢复、青藏高原的特殊 生境、全球变化下的中国陆地生态系统响应、植物对 环境污染的修复作用等，二要保证研究手段的不断 更新，在国际高水平学术刊物上发表高质量的论文。 例如，所使用的仪器必须是国际同行所认可的，一些 质量和信誉很差的仪器所获得的数据在有影响的国 际专业刊物上发表的机会很小。加强我国的植物生 理生态学研究, 既能满足解决国民经济发展中的实 际生态问题的需求，又可使中国植物生理生态学研 究在国际大舞台上有 表演”的机会。在今后的国际 学术竞争中，我们是依然喟 配角”,还是争取喟 主 角”取决于我们这一代人的努力。

\section{参 考 文 献}

Bates, C. G. \& J. Jr. Roeser. 1928. Light intensities required for growth of coniferous seedlings. American Journal of Botany, 15: $185 \sim 194$.

Billings, W. D. 1985. The historical development of physiological plant ecology. In: Chabot, B.F. \& H. A. Mooney eds. Physiological ecology of north American plant communities. New York: Chapman and Hall. $1 \sim 15$.

Blackman, F. F. \& G. L. Matthaei. 1905. Experimental studies on vegetable assimilation and respiration. IV. A quantitative study of carbon dioxide and assimilation and leaf temperature in natural illumination. Proceedings of Royal Society Bulletin, 76: $402 \sim 460$

Blackman, F. F. 1905. Optima and limiting factors. Annuals of Botany, 19: $281 \sim 295$.
Boyle, J. S. 1661. The sceptical chymist. London.

Briggs, G. E. 1922. Experimental researches on vegetable assimilation and respiration. XVI. The characteristic activity resulting from deficiency of nutrient salts. Proceedings of Royal Society Bulletin, 94: $20 \sim 35$.

Briggs, G. E., R. Kidd \& C. West. 1920. Quantitative analysis of plant growth. Annals of Applied Biology, 7: $101 \sim 223$.

Chang, J. (常杰), G. Chen (陈刚) \& Y. Ge (葛溁). 1995. The segregation character and modeling of plant structure. Hangzhou: Hangzhou Universty Press. (in Chinese)

Chien, C. S. \& W. J. Osterhout. 1917. Peculiar effects of barium, strontium and cerium on Spirogyra. Botanical Gazette, 63: $406 \sim 409$.

Clements, F. E. 1907. Plant physiology and ecology. New York: Holt.

Cui, C. (崔 $\square$ ). 1954. The distribution of zinc and ABA in plant and their relation with growth. Acta Botanica Sinica (植物学 报), 3:31 36. (in Chinese)

Daubenmire, R. F. 1974. Plant communities. New York: Haper \& Row.

de Wit, C. T. 1978. Simulation of assimilation, respiration and transpiration of crops. Wageningen Pudoc Scientific Publishers.

Edwards, G. \& D. Walker. 1983. C $3, C_{4}$ : mechanisms, and cellular and environmental regulation of photosynthesis. Oxford, London, Edinburgh, Boston, Melbourne: Blackwell Scientific Publications.

Farquhar, G. D., S. von Caemmerer \& J. A. Berry. 1980. A biochemical model of photosynthetic $\mathrm{CO}_{2}$ assimilation in leaves of $\mathrm{C}_{3}$ species. Planta, 149: $78 \sim 90$.

Farquhar, G.D. \& S. von Caemmerer. 1982. Modeling of photosynthetic response to environmental conditions. In: Lange, O. L., P.S. Noble, C. B. Osmond \& H. Ziegler eds. Encyclopedia of plant physiology, Vol. 12B. Physiological plant ecology, Vol. II. Berlin: Springer. $550 \sim 587$.

Gates, D. M. 1962. Energy exchange in the biosphere. New York: Haper \& Row.

Haberlandt, G. 1884. Physiologische pflanzenanatomie. Leipzig: Engelmann.

Haekel, E. 1866. Generelle morphologie der organismen. Jana. Hales, S. 1727. Vegetable staticks. London: W. \& J. Innys.

Holmgren, P. , P. G. Jarvis \& M. S. Jarvis. 1965. Resistances to carbon dioxide and water vapour transfer in leaves of different plant species. Physiologia Plantarum, 18: $557 \sim 573$.

Hoover, W. H. , E. S. Johnston \& F. S. Brackett. 1933. Carbon dioxide assimilation in a higher plant. Smithsonian Misc. Collection, 87(16): $1 \sim 31$.

Jiang, G. M. (蒋高明). 2001. Review on some hot topics towards the researches in the field of plant physioecology. Acta Phytoecologica Sinica (植物生态学报), 25: $514 \sim 519$. (in Chinese with English abstract)

Kortschack, H. P., E. E. Hartt \& G. O. Burr. 1965. Carbon dioxide fixation in sugarcane leaves. Plant Physiology, 40: 209 $\sim 213$.

Larcher, W. (translated by Li, B. (李博)). 1980. Plant physioecology (1st ed). Beijing: Science Press.

Larcher, W. (Translated by Zai, Z. S. (翟志席)). 1997. Plant ecophysiology. (5th ed). Beijing: Science Press. 
Larcher, W. 1975. Physiological plant ecology. Berlin: Springer. Li, J. T. 1929. The immediate effect of light on the rate of photosynthesis. Annals of Botany, 43: $587 \sim 601$.

Li, J.T. (李继侗). 1986. On constitution of climate. In: Committee of Professor Li's Works (李继侗文集编委会) ed. Bulletin of professor Li's works. Beijing: Science Press. 133 145 . (in Chinese)

Li, J.T. (李继侗). 1958. The development of plant geography, plant ecology and botanicalgraphy. Beijing: Science Press. (in Chinese)

Liebig, J. 1862. Die naturgesetze dies feldbaues. Vieweg: Braunschweig.

Loo, T. I. 1927. The influence of hydrogen ion concentration on the growth of the seedlings of some cultivated plants. Botanical Magazine, $41: 33 \sim 41$.

Loomis, W. E. 1960. The discovery of photosynthesis. In: Ruhland, W. ed. Encyclopedia of plant physiology. (Vol. V, Part 1). Berlin, Göttingen, Heidelberg: Springer-Verlag. $84 \sim 114$.

Ludlow, M. M.\& G. L. Wilson. 1971. Photosynthesis of tropical pasture plants. II. Temperature and illuminance. Australian Journal of Biological Science, 24: $1065 \sim 1075$.

Maximov, N. A. 1929. The plant in relation to water. London: Allen \& Unwin.

Monteith, J. L. 1965. Evaporation and environment. Symposium of the Society for Experimental Biology.
Pfeffer, W. 1900. The physiology of plants (English translation). Oxford: Clarendon. German first edition 1881.

Schimper, A. F. W. 1898. Pflanzengeopraphie auf physiologische grundlage. Jena: Fischer. (English translation, 1903. Oxford: Clarendon Press)

Tang, Y.W. (汤玉玮) , J. S. Ni (倪晋山)，C. Z. Jin (金成 忠)，H. C. Lei (雷宏口), J. N. Shi (施教耐),Z. R. Zheng (郑泽荣) \& J. L. Zhang (张静兰). 1955. The need of wheat for mineral elements in different growth period. Acta Botanica Sinica (植物学报), 4:137 152. (in Chinese with English abstract)

Tang P. S. (汤佩松)，Y. L. Dai (戴云玲) \& J. G. Li (李佳 格). 1956. Respiration and its physiological adaptation in rice seedling. Acta Botanica Sinica (植物学报), 5:377 398. (in Chinese with English abstract)

Tang, P. S. \& J. S. Wang. 1941. A thermodynamic formulation of the water relations in an isolated living cell. Journal of Physical Chemistry, 45:443 453.

von Humboldt, A. 1805. Ideen zu Einen Geographie der Pflanzen. Tubingen: Cotta.

Warming, E. 1891. De Psammofile vegetationer in Danmark. Foren: Vid. Medd.

Woodward, J. 1699. Thoughts and experiments on vegetation. Philosophical Transactions of Royal Society London, 21: 382 398 . 